\title{
Demo: Composing Services in 5G-TRANSFORMER
}

\author{
Jorge Baranda, Josep Mangues-Bafalluy, Luca Vettori, Ricardo Martínez, Giada \\ Landi $^{\star}$, Kiril Antevski ${ }^{\dagger}$ \\ Centre Tecnològic de Telecomunicacions de Catalunya (CTTC/CERCA), Castelldefels, Spain; ${ }^{\star}$ Nextworks, \\ Italy; ${ }^{\dagger}$ Universidad Carlos III de Madrid, Spain \\ \{baranda,josep.mangues,lvettori,rmartinez\}@cttc.cat;g.landi@nextworks.it;kantevsk@pa.uc3m.es
}

\begin{abstract}
5G mobile networks need flexilibity, dynamicity and programmability to satisfy the needs of vertical industries. In such context, network services can be designed as integral units to be dynamically grouped among them to create tailored complex composite services or to allow the combination of services in a context of network slicing. In this demonstration, we present the service composition capabilities of the 5G-TRANSFORMER platform. In particular, we will show the instantiation of a composite network service using a previously instantiated service. When terminating the composite network service, the initial instantiated network service resumes its operation without disruption.
\end{abstract}

\section{CCS CONCEPTS}

- Networks $\rightarrow$ Network experimentation; Programmable networks; Network management;

\section{KEYWORDS}

Service composition, End-to-End service orchestration, NFV, SDN, 5G mobile transport

\section{ACM Reference Format:}

Jorge Baranda, Josep Mangues-Bafalluy, Luca Vettori, Ricardo Martínez, Giada Landi ${ }^{\star}$, Kiril Antevski ${ }^{\dagger}$. 2019. Demo: Composing Services in 5G-TRANSFORMER. In Proceedings of ACM International Symposium on Mobile Ad Hoc Networking and Computing (MobiHoc'19). ACM, New York, NY, USA, 2 pages. https://doi.org/XX.XXX/XXX_X

\section{INTRODUCTION}

In the $5 \mathrm{G}$ context, network services (NSs) can be designed as integral units that work on their own, but they can also be dynamically grouped with other services to create more complex and tailored offerings. This allows better adapting to the needs of a variety of vertical industries, which may be

Permission to make digital or hard copies of part or all of this work for personal or classroom use is granted without fee provided that copies are not made or distributed for profit or commercial advantage and that copies bear this notice and the full citation on the first page. Copyrights for thirdparty components of this work must be honored. For all other uses, contact the owner/author(s).

MobiHoc'19, fuly 2-5 2019, Catania, Italy

() 2019 Copyright held by the owner/author(s).

ACM ISBN XXX-XXXX-XX-XXX/XX/XX.

https://doi.org/XX.XXX/XXX_X sharing the same network. To be able to realize this vision, the $5 \mathrm{G}$ network, by means of its control and management framework, must offer the required functionality. In that sense, service composition is a fundamental mechanism to enable the instantiation of NSs in the form of a dynamic puzzle. In this demonstration, we will show the capabilities of the 5G-TRANSFORMER platform[1], specially the 5GT-Service Orchestrator (5GT-SO), to manage end-to-end composite network services deployed on a complex transport network including heterogeneous transport technologies (wireless, optical) and several multi-PoP (Point of Presence) computing resources. In particular, we will demonstrate the instantiation/termination of a composite network service emulating a monitoring backend application, which uses a previously instantiated NS emulating a vEPC to dynamically build a more complex one. To the best of our knowledge, this is the first operational demonstration of a composite network service deployment sharing a previously deployed network service.

\section{SYSTEM ARCHITECTURE}

Figure 1 presents the system under demonstration. The value proposition of this demonstration lies in the 5GT-SO module, which has been enhanced with a hierarchy (parent-child) of service orchestrator engine (SOE) modules to handle composite network service requests. The SOE parent (SOEp) is in charge of analysing the incoming request and orchestrates all the required operations in case of a composite service. Thanks to the modular architecture of the 5GT-SO module, the SOEp relies on the SOE child to perform the instantiation of each of the different nested NS in a composite NS, following the original orchestration logic explained in [3] using ETSI NFV IFA 014, 011, 013 specifications. The 5G-MTP module is the coordinator of the different managers present at the underlying transport network providing storage, computing and networking resources. In particular, storage and computing resources are provided by two PoPs managed by different instances of Openstack software, while networking resources at the transport network are coordinated by a hierarchy of SDN controllers using Control Orchestration Protocol (COP) [2]. The transport network combines different transport technologies. At the edge, forwarding elements use wireless mmWave (IEEE 802.11ad) and WiFi (IEEE 802.11ac) 


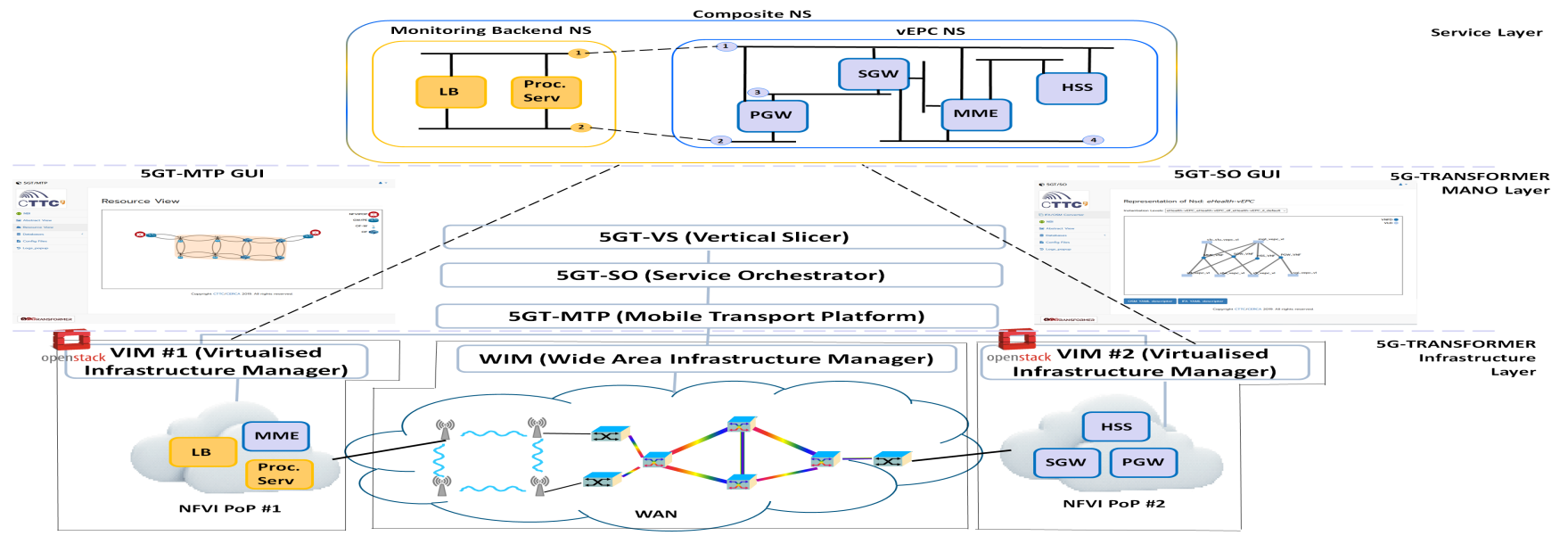

Figure 1: Deployment of a composite NS over the 5G-TRANSFORMER platform

links. At the core domain, the system counts with a multilayer optical transport network, as depicted in Figure 1.

\section{DEMONSTRATION}

In this demonstration, we show the automated end-to-end orchestration of a composite NS which is sharing resources with a previously instantiated NS on a multiPoP infrastructure connected by the transport network described previously. The composite NS consists of two nested NSs. First, a NS emulating a Virtualized Evolved Packet Core (vEPC). This vEPC NS consists of four VNFs, namely MME, HSS, S-GW and P-GW. The second nested NS is a monitoring backend NS consisting of two VNFs, namely a load balancer and a processing server. The main steps of the demonstration are: 1) A request of a single NS arrives to the 5GT-SO from the 5GT-VS. It asks for the deployment of the simulated vEPC NS. The 5GT-SO analyses the request and proceeds with the deployment workflow of a single NS, as explained in [3].

2) A request of a composite NS consisting of two different nested services (a vEPC and a monitoring backend) arrives to the 5GT-SO. In this request, there is a reference to an already deployed network service instance. The 5GT-SO analyses the request, checks which are the nested NSs of the composite network service and identifies to what part of the composite network service, the received reference points to.

3) Based on the information extracted in step 2), the 5GT-SO proceeds to instantiate the nested NSs which is not currently deployed according to the deployment workflow of a single NS, as it is done in step 1).

4) Once all the different nested NS of the composite are instantiated, the 5GT-SO triggers the creation of the internested NS connection/s according to the description provided in the composite network descriptor. 5GT-SO performs an appropriate management of its databases to track the information and resources related to both the composite and the nested service instances.

In the second part of the demostration, we show the termination of the composite NS. In this case, the 5GT-SO will proceed to remove the resources associated to the composite NS (i.e. second nested NS) without disrupting the operation of the initially instantiated simulated vEPC network service. Several graphical user interfaces (GUIs) show information about the different components of the 5G-TRANSFORMER system, as depicted in Figure 1. For instance, the GUI of the 5GT-SO module shows the structure of the different deployed NS and the GUI of the 5GT-MTP module shows the underlying networking and compute infrastructure together with the resources used by the instantiation of the different NSs.

\subsection{Requirements}

The demonstration will be launched from a laptop and executed remotely at the CTTC premises, hence, requiring stable Internet access. A table will be required to place the laptop and one or two large screens to show the available GUIs and access to a power outlet to plug these elements. A wall or stand to mount a poster will help to explain the demo to the audience. A demo run is about 20 minutes.

\section{ACKNOWLEDGMENTS}

This work has been partially funded by the EC H2020 5GTransformer Project (grant no. 761536), by MINECO grant TEC2017-88373-R (5G-REFINE) and Generalitat de Catalunya grant 2017 SGR 1195.

\section{REFERENCES}

[1] 2018. EU H2020 5G-Transformer, 5G Mobile Transport Platform for Verticals. http://5g-transformer.eu/.

[2] J. Baranda et al. 2018. Orchestration of end-to-end network services in the 5G-Crosshaul multi-domain multi-technology transport network. IEEE Comm. Mag. Net. and Service Man. Series 57 (July 2018).

[3] J. Mangues et al. 2019. 5G-TRANSFORMER Service Orchestrator: design, implementation, and evaluation. In Submitted to 28th European Conf. on Net. and Comms. (EUCNC 2019), 18-21 June, Valencia (Spain). 